See discussions, stats, and author profiles for this publication at: https://www.researchgate.net/publication/334290557

\title{
SHMnet: Condition Assessment of Bolted Connection with Beyond Human-
} level Performance

\section{Preprint · July 2019}

DOI: $10.13140 / R G .2 .2 .17510 .57929$

CITATION

1

3 authors:

(2)

Tong Zhang

Peng Cheng Laboratory

25 PUBLICATIONS 507 CITATIONS

SEE PROFILE

ค) Ying Wang

University of Surrey

62 PUBLICATIONS 537 CITATIONS

SEE PROFILE

Some of the authors of this publication are also working on these related projects:

An integrated physics-based and data-driven approach to structural condition identification View project

Intelligent Fetal Imaging and Diagnosis Project: clinical View project

\section{READS}

1,001

.7. Suryakanta Biswal

University of Surrey

11 PUBLICATIONS 37 CITATIONS

SEE PROFILE 


\title{
SHMnet: Condition Assessment of Bolted Connection with Beyond Human-level Performance
}

\author{
Tong Zhang ${ }^{1}$; Suryakanta Biswal ${ }^{2}$; Ying Wang ${ }^{2 *}$
}

\begin{abstract}
Deep learning algorithms are transforming a variety of research areas with accuracy levels that the traditional methods cannot compete with. Recently, increasingly more research efforts have been put into the structural health monitoring (SHM) domain. In this work, we propose a new deep convolutional neural network, namely SHMnet, for a challenging structural condition identification case, i.e. steel frame with bolted connection damage. We perform systematic studies on the optimization of network architecture and the preparation of the training data. In the laboratory, repeated impact hammer tests are conducted on a steel frame with different bolted connection damage scenarios, as small as one bolt loosened. The time-domain monitoring data from a single accelerometer are used for training. We conduct parametric studies on different sensor locations, the quantity of the training data sets, and noise levels. The results show that the proposed SHMnet is effective and reliable with at least four independent training data sets and by avoiding vibration node points as sensor locations. Under up to $60 \%$ addictive Gaussian noise, the average identification accuracy is over $98 \%$. In comparison, the traditional methods based on the identified modal parameters inevitably fail due to the unnoticeable changes of identified natural frequencies and mode shapes. The results provide confidence in using the developed method as an effective structural condition identification framework. It has the potential to transform the SHM practice.
\end{abstract}

\section{Keywords}

Deep learning, Condition identification, CNN, Data preparation, Bolted connection

\section{Introduction}

It is undeniable that civil infrastructure systems are critically important to modern society. The costs to maintain and repair these infrastructure systems over their long service lives are significant. Among them, bolted connections have been used in metallic structures for many years and now become increasingly popular due to the recent development of off-site modular constructions (Lacey et al. (2018)). Connection stiffness is a critical factor to determine the overall performance of metallic structures. In the design phase, bolted connections are normally assumed either fixed or pinned. However, in the service stage, almost all the connections are between these two idealised conditions, i.e. semi-rigid, which may lead to an obvious difference between numerically simulated results and actual structural performance. Further, bolted connections inevitably deteriorate over time like other structural components, due to fatigue, corrosion, and other factors. These may affect the structural performance significantly. For example, even one damaged bolt may lead to significant increase of the stresses in the adjacent bolts (Blachowski and Gutkowski (2016)). Therefore, the condition assessment of bolted connections in metallic structures is needed for the effective maintenance and operation of these structures.

Different approaches have been used to identify conditions for bolted connections, which can be categorised as local and global. The local methods, or termed as non-destructive evaluation methods, include relative displacement sensors
(Li and Hao (2016)), vision-based methods (Cha et al. (2016)), acoustoelastic effect based methods (Wang et al. (2013)), piezoelectric impedance method (Shao et al. (2016)), and piezoelectric active sensing method (Wang et al. (2013, 2017)). Although these methods have achieved very good measurement results, they are not suitable for the practical application to civil infrastructure systems for the following two reasons: 1) the sensing systems are normally complicated and expensive than the normal vibration-based structural health monitoring (SHM) system; 2) the methods usually require the sensors to be located close to the targeted elements. In contrast, the global methods, i.e. vibrationbased methods, can overcome the above two difficulties. A number of different approaches (Yang et al. (2013); He and Zhu (2011); Yun et al. (2001)) have been proposed, which demonstrated the effectiveness of the global methods.

Nevertheless, most of the existing vibration-based methods can only detect damage at the connection level (i.e. all bolts damaged/loosened at one joint), using the traditional

\footnotetext{
${ }^{1}$ School of Biomedical Engineering and Imaging Science, King's College London, UK

${ }^{2}$ Department of Civil and Environmental Engineering, University of Surrey, UK
}

\section{Corresponding author:}

Ying Wang, Department of Civil and Environmental Engineering, Faculty of Engineering and Physical Sciences, University of Surrey Guildford, GU2 7XH, the United Kingdom

Email: ying.wang@surrey.ac.uk 
physics-based methods. One important potential reason is that these methods usually use the modal parameters extracted from vibration signals as the features, i.e. natural frequencies and mode shapes, and/or their derivatives. However, these features may not be sensitive to small damage, because they inevitably lose subtle (transient and/or nonlinear) information compared with that from original time domain data. Another potential reason is that it is relatively hard to simulate actual behaviours of the connections, especially the damping effects.

Data-driven approaches provide an alternative path to interpret vibration-based monitoring data and have recently attracted increasing research attention. With sufficient training data sets, the supervised (or semi-supervised) learning schemes can generate and optimise the features automatically so as to enhance their effectiveness. Thus, these methods may achieve better structural condition identification results than traditional methods, while computational costs are significantly reduced. For example, Ay et al. (2019) developed a data driven method which used the probability distribution of decay rate to correlate the overall damping effects with the connection damage, and achieved better results than the traditional modal identification methods.

However, the existing data-driven methods faces two main challenges. Firstly, they often lack the complexity embedded in numerous and diverse scenarios in real engineering structures, considering different possible conditions, environmental factors, nonlinear structural responses and loading histories. Secondly, they almost always suffer from the lack of "sufficient" training data. A question associated to this is how many training data are sufficient for the reliable training of a machine learning algorithm.

In this study, we aim to overcome the above-mentioned challenges by 1) proposing a novel deep learning algorithm, SHMnet; and 2) optimising its performance, by performing parametric studies for data preparation. In the rest of this paper, we firstly review the related works and highlight our contribution. Secondly, we describe the proposed methodology, i.e. SHMnet and its training data preparation scheme. Thirdly, we present a laboratory case study on a scaled steel frame structure with various bolted connection damage scenarios (from one bolt loosening to all four bolts loosening). Fourthly, we perform a series of parametric studies, and evaluate the performance of the proposed method in comparison with the traditional modal-based methods. Finally, based on the results and discussions, conclusions and future recommendations are presented.

\section{Related Works}

From machine learning perspective, the structural condition identification is a typical pattern recognition process (Farrar and Worden (2013)). Given sufficient monitoring data sets $X$ including their corresponding pattern label set $Y$ and a proper machine learning algorithm, the inherent features of of the data sets can be obtained through a training process. Based on the trained model, the feature of a new monitoring data set with unknown condition, $x$, can be easily computed. By comparing the obtained feature with those in the training data sets, the one with least difference can be regarded to have the same pattern (structural condition) as $x$. Thus, $x$ will be assigned with the label of this data set. Based on this process, it is not difficult to infer that the performance of data-driven approaches is controlled by the effectiveness of the machine learning algorithm and the quality and quantity of training data sets.

\section{Deep learning}

Recently developed deep learning method enables the modelling of complexity through multiple learning layers. It has attracted significant scientific interest and shown extraordinary effectiveness with beyond human level accuracy in different domains, e.g. image understanding, language processing, and the game of Go (LeCun et al. (2015); Silver et al. (2016)). It constructs a multi-layer neural network to learn features of the data with various levels of inherent patterns. A typical deep learning model, convolutional neural network $(\mathrm{CNN})$, includes convolutional layers, rectified linear unit (ReLU) layers, pooling layers, dropout layers and fully connected layers. The convolutional layer, consisting of a set of kernels, is the core building block of a CNN. It essentially computes linear operations (multiplications and summations). The ReLU layer aims to introduce nonlinearity to a system, by applying the function $f(x)=\max (0, x)$ to all of the values from previous layer. The pooling layer can perform down-sampling operations. The most popular option, i.e. the $\max$ pooling method, divides the input data into a set of non-overlapping subsets and, for each sub-set, outputs the maximum. As a very popular option (other layers being essential), the dropout layer is usually used as an empirical regularization method. Aiming to at least partially solve the problem of over-fitting, this layer drop out a random set of nodes in that layer by setting them to zero. Lastly, the fully connected layer takes the output from the previous layers and outputs an $N$ dimensional vector, where $N$ is the number of classes that the algorithm needs to choose from.

There are many CNN models available, while the network architecture is a key factor influencing the performance of a model. For example, the Alex-Net (Krizhevsky et al. (2012)) was arguably regarded as the first breakthrough in deep learning, which significantly outperformed all the prior competitors and won the ImageNet challenge in 2012 by reducing the top-5 error from $26 \%$ to $15.3 \%$. Its network architecture is a good prototype for other applications.

\section{Application of deep learning to SHM}

In the SHM domain, the application of deep learning algorithms has gained limited yet fast increasing research attention. It has shown the great potential to solve the first challenge introduced above.

The current studies on the application of deep learning in the SHM domain can be classified into two categories. The first approach is a direct adaptation from computer vision application, i.e. detecting different structural conditions based on the analysis of images, either the photos of a particular structural location (Cha et al. (2017); Zhang et al. (2019)) or the vibration time histories under different scenarios (Bao et al. (2018)). The second approach is to identify structural conditions through a supervised (or semisupervised) learning process, based on the training data sets 
of vibration responses. The latter are more promising as they can potentially construct more effective features, and are more compatible and comparable with the physics-based methods. For example, Abdeljaber et al. (2017) developed an in-house MATLAB program based on 1D convolutional neural networks (1D-CNN), with successful application to QU grandstand simulator (Abdeljaber et al. (2017)) and IASC-ASCE SHM benchmark problem (Abdeljaber et al. (2018)). Pathirage et al. (2018) proposed an autoencoder based framework for structural damage identification and verified the framework on an eight-story steel frame structure. Further, Pathirage et al. (2019) proposed a deep sparse autoencoder framework, and validated it on a numerical case of a steel frame structure and an experimental case of a prestressed concrete bridge.

Indeed, the architectures and parameters of deep learning algorithms may vary for different problems. For the popular CNN models, number of different layers, number of kernels, max pooling shape, dropout rate, and other parameters are variable. Although it is expected that the network will perform better with deeper and wider convolution layers, it may suffer from the over-fitting problem. Based on the authors' knowledge, the existing literature with SHM applications have not discussed how to optimise the network architecture and how to set the parameters.

The performance of a deep learning algorithm not only depends on the network architecture, but also relies on the quality and quantity of the training data. The quantity of training data are normally controlled by the experimental design and the repeated test numbers, while the quality of training data are affected by many factors, including the test method, data type, sensor location, etc. Zhang and Wang (2019) discussed the effect of the quantity of repeated tests on the performance of structural identification, based on a case of steel truss bridge with connection damage. However, systematic studies on the effects of different abovementioned factors were rarely discussed in the existing literature.

\section{Contribution of this work}

Realising above research gaps, this work focuses on the development of a novel deep neural network, i.e. SHMnet. We select a state-of-the-art network configuration and make necessary adjustments to suit the case considered in this study. Further, we perform parametric studies on different influencing factors, i.e. 1) different noise levels for data augmentation; 2) different sensor locations; 3) different quantities of training data sets. Based on the results, an optimised network architecture and the training data preparation scheme is obtained. We further test the robustness of the proposed SHMnet by adding up to $100 \%$ random Gaussian noise levels of noise to the test data, and compare its performance with traditional modal-based methods.

\section{Methodology}

\section{SHMnet}

In this study, we constructed the SHMnet based on the Alexnet for better performance and efficient training and testing, by making the following adjustments.

1. Since the vibration monitoring data are intrinsically time-series, i.e. one-dimensional (1D), we adjusted all the two-dimensional layers to $1 \mathrm{D}$.

2. We selected the most popular optimisation algorithm, Adam (Kingma and Ba (2014)), for efficient training.

3. We set and adjusted the network parameters, including the number of convolutional layers, the number of filters, max pooling shape, and dropout rate, based on empirical results. For example, we constructed the network with three convolutional layers, and then compared its performance with those of deeper networks. The results showed that the network is tend to be over-trained with deeper (more than three) convolutional layers, which is consistent with the previous findings in 1D-CNN Abdeljaber et al. (2017). To save the computational resources and avoid further investigations on the early stop criteria, we simply set the network with three convolutional layers, which gives the best results. The SHMnet framework is shown in Figure 1 , and the details of the network architecture and the parameters are presented in Table 1. The input of SHMnet is the vibration responses, while the output is a label of structural condition which associated to the particular vibration response. SHMnet itself contains two main parts: feature extraction and classification. The feature extraction part consists of three 1D convolutions layers with kernel size 7, 5 and 3, each followed by an non-linear ReLU activation (Nair and Hinton (2010)), and max pooling with kernel size 3 and stride of 2 . The classification part involves two dropout layers Srivastava et al. (2014) each with a rate of 50\%, two ReLU layers and three dense/fully connected layers which finally propagate the learned features to the total number of output classes which can be adapted to different cases. In this study, since 11 scenarios are considered, we set this number as 11. Based on empirical results and previous studies, we set the learning rate to 0.0001 and the number of training epochs as 1000 .

For easy adaptation and further implementation in other data sets, the program is written in a popular deep learning platform, PyTorch (https://pytorch.org) and is available for downloading at https://github.org.

\section{Training data and data preparation}

In this work, the repeated vibration test data in time domain, obtained from a single accelerometer under different scenarios, are used as the training data.

The training data can be obtained either experimentally or numerically. Compared with the use of numerical simulation data (Wang and Hao (2013)), the use of test data can avoid the modelling error, and thus the test is selected.

Regarding the test method, ambient vibration, shaker, and impact hammer, are the most popular choices for modal testing (Ewins (2000)). Ambient vibration is more suitable for real structures under various loads, e.g. traffic loads, environmental loads, seismic loads, etc. The latter two are more suitable for laboratory testing. An electrodynamic 


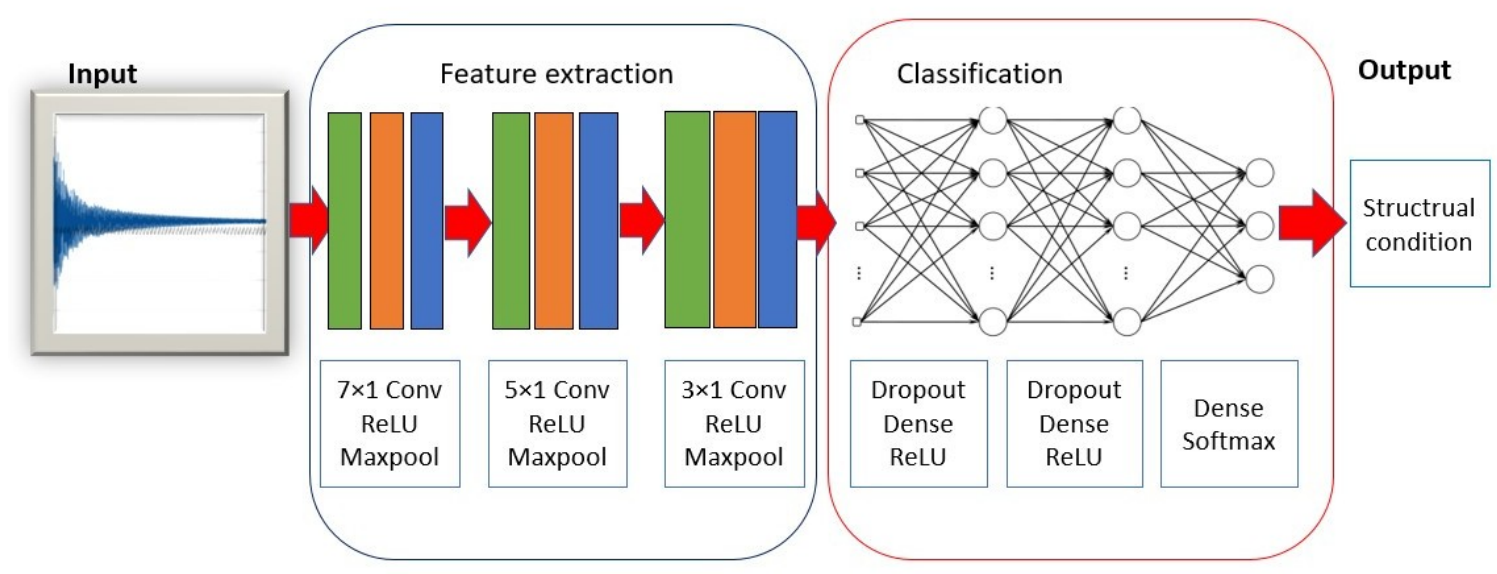

Figure 1. Structural Health Monitoring network: SHMnet

shake normally conducts forced-vibration test either through sweeping individual vibration modes of interest or through random vibration with different frequency components. In contrast, an impact hammer produces a single, high resolution, transient response waveform that can generate vibration data with a fairly wide frequency band. Therefore, for the laboratory tests in this study, the impact hammer method is selected.

Regarding the data type, compared to the commonly used frequency domain methods (e.g. Pathirage et al. (2018)), time domain methods (e.g. Abdeljaber et al. (2017)) essentially contain all the vibration information, including non-linear and transient effects which are often missed by the former, while they are more computationally efficient, as there is no need for domain transformation. Thus, time domain data will be used directly as training data in this work.

After determining the test method and the data type, we still need to perform the following three parametric studies for data preparation:

\section{Table 1. SHMNET NETWORK ARCHITECTURE}

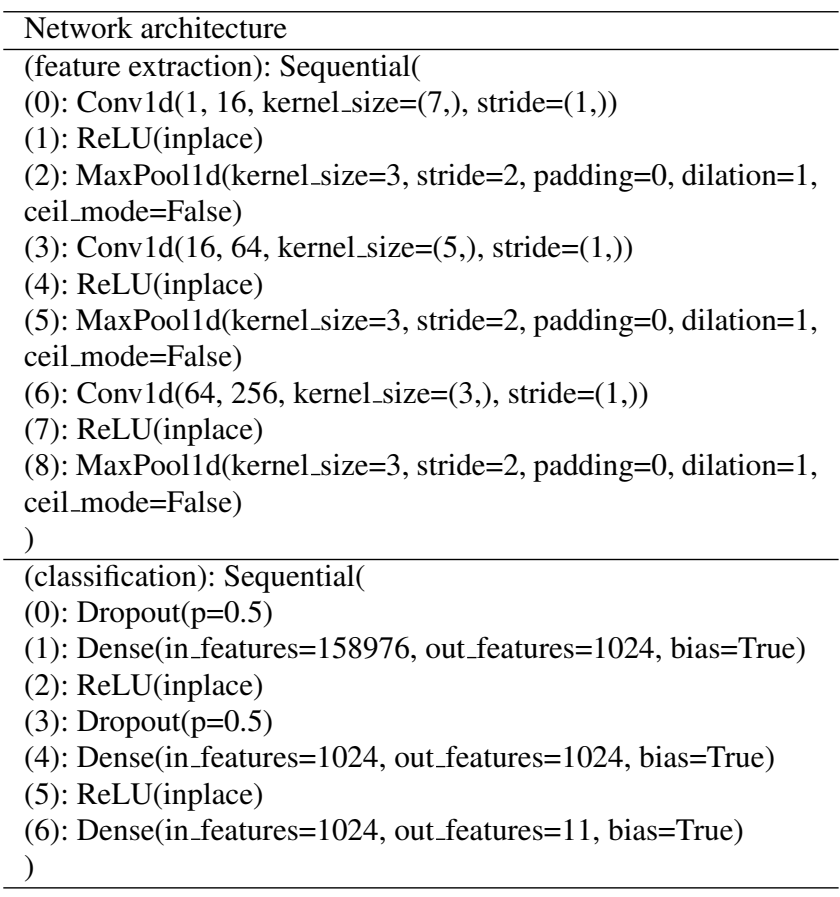

1. We will augment the training data by adding Gaussian noise to the original test data. This aims to improve the training and test accuracy and to mitigate the over-fitting problems. The algorithm performance at different levels of noise will be evaluated.

2. To investigate the effects of sensor locations, we record the data from a total of six accelerometers. Their performance as training data will be compared.

3. To evaluate the effects of the quantity of training data, we will compare the algorithm performance on the use of different quantities of repeated test results.

\section{Experimental study: a frame structure with bolt connection damage}

In order to evaluate the effectiveness of the proposed methodology on a challenging case, a steel frame with connection damage as small as one bolt loosening is selected in this study.

\section{Test structure}

A single bay single storey steel frame was constructed in the laboratory, as shown in Figure 2. The beam with a $2.45 \mathrm{~m}$ length and the UB $127 * 76 * 13$ section was connected to the columns with a $1.5 \mathrm{~m}$ height and the UC $152 * 152 * 23$ section, by using four gusset angles with the USA $100 * 65 * 8$ section and eight $10 \mathrm{~mm}$ pretension bolts. A pretension torque of $55 \mathrm{Nm}$ was applied to the bolts using a torque wrench, to represent the fully tightened condition. To provide the fixed supports at the bottom, the columns were welded to the base plates which were then bolted to the strong floor. The geometric details of the frame and bolted connection details with specified bolt numbers are shown in Figure 3.

\section{Test setup}

The modal test was carried out using an instrumented impulse hammer (Model 2304) and six accelerometers (Model 256-100) from Meggitt Endevco Sensing Systems. The maximum impulse capacity of the instrumented hammer is $35584 \mathrm{~N}$, with a sensitivity of $0.023 \mathrm{mV} / \mathrm{N}$. The accelerometers are rated at $100 \mathrm{mV} / \mathrm{g}$ with an acceleration measurement range of $50 \mathrm{~g}$. To achieve data acquisition, the impact hammer and accelerometers were connected to 


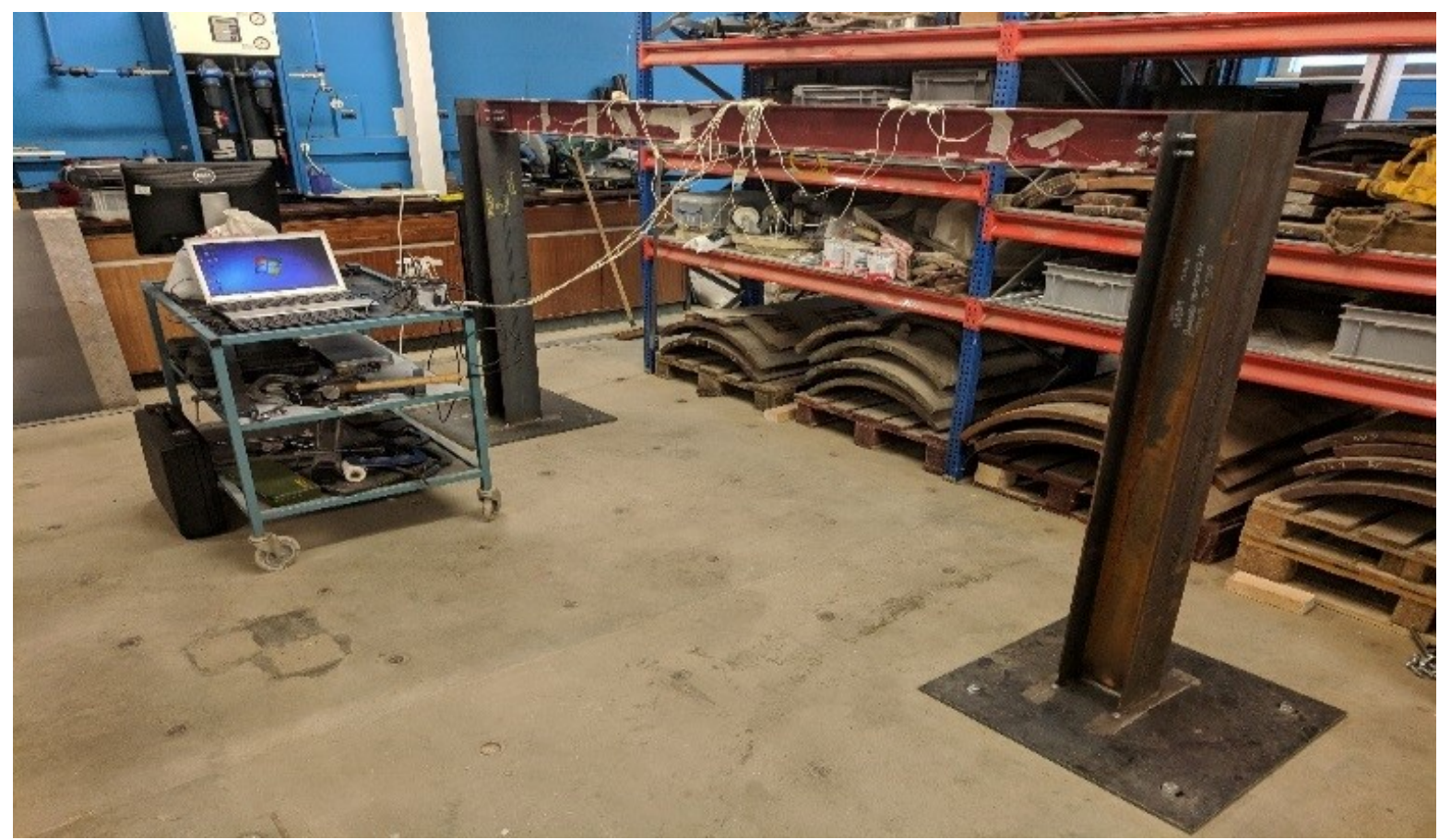

Figure 2. Steel frame in the laboratory

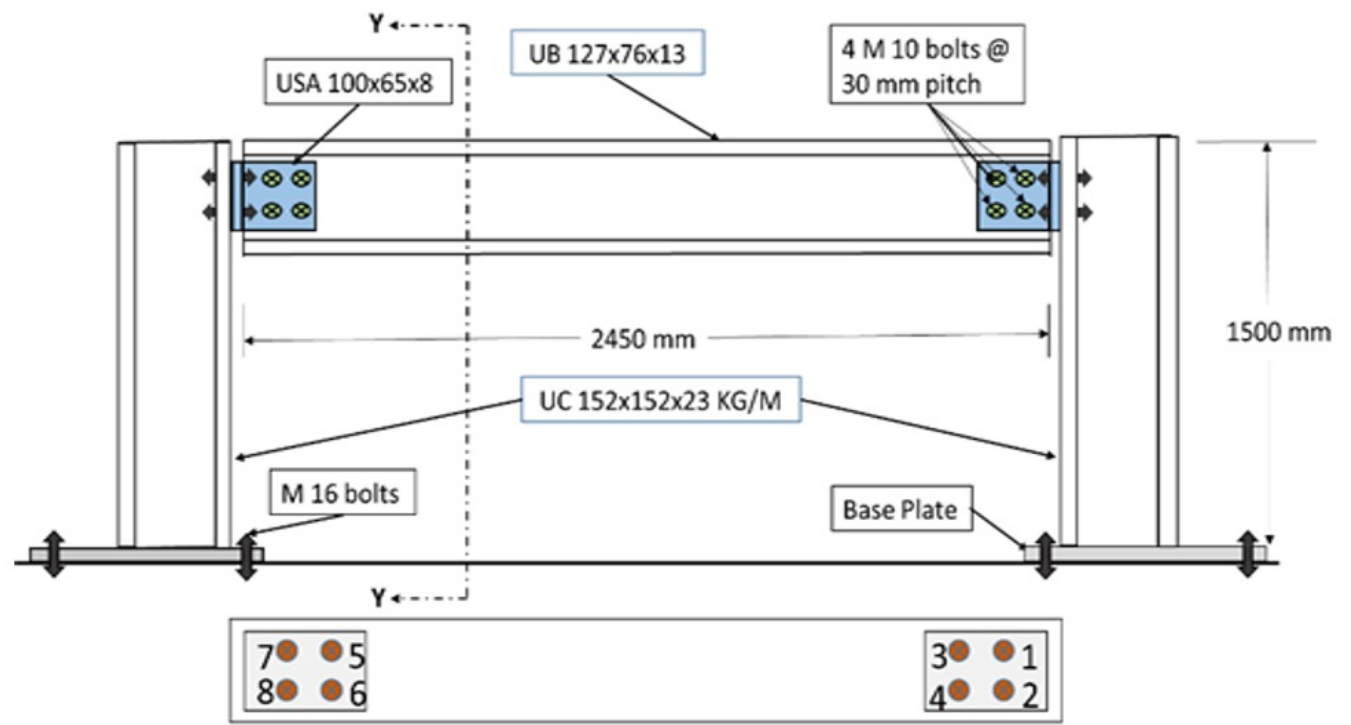

Figure 3. Test frame details

a sound and vibration input module, National Instruments 9234, within the chassis, National Instruments cDAQ-9178.

The impact point was selected close to but not at the exact quarter point of the beam, to avoid the vibration node locations. The locations of the accelerometers were determined by using a recently proposed algorithm of damage measurability (Biswal and Wang (2019)). A line diagram of the portal frame showing locations of the accelerometers and the impact hammer is shown in Figure 4.

Six data samples from six accelerometers can be obtained per strike. Based on our preliminary study on this structure, the sampling rate is set as $4096 \mathrm{~Hz}$, which is more than enough to cover the first three natural frequencies of the test structure (the higher modes results become noisy). The length of each data sample is taken as 10,000 , which is equivalent to 2.34 seconds. This allows the accelerometers to record the transient vibration behaviours of different points on the structure, from the starting of the strike, until the vibration amplitude is very small.

\section{Test scenarios}

Ideally, the experimental design should cover all the possible damage scenarios. However, this is almost always impossible, because the possible structural damage scenarios are essentially infinite, while the resources for experiments are limited. In such case, the classes of the training data can be systematically designed according to three levels: damage type, location and severity, e.g., Wang and Hao (2013). 


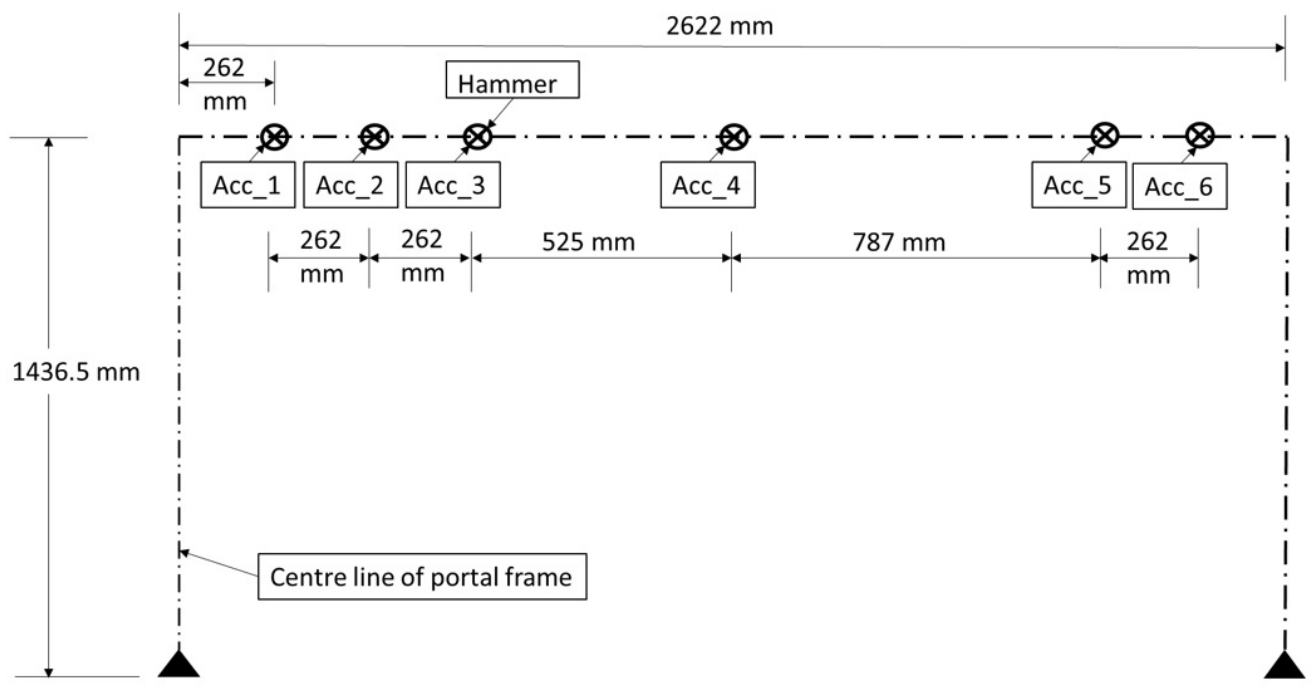

Figure 4. The location of sensors and impact hammer for the modal testing

In this work, ten damage scenarios plus the intact scenario are considered, as listed in Table 2. For scenarios 1-3, we examine the conditions with only one bolt loosened at different locations. For scenarios 4-6, we consider the conditions with two bolts loosened with different combinations. For scenarios 7 and 8, we address two bolts loosened on both sides of the beam. For scenario 9, we examine the condition with three bolts loosened. Therefore, the first nine scenarios address the conditions that only a part of bolted connections are loosened. Indeed, most existing studies deal with the identification of the whole connection loosened, which is represented in this work as scenario 10 with all four bolts loosened.

In order to generate enough data to investigate the effects of the quantity of training data, ten impact hammer tests were performed for each damage scenario. Therefore, a total of 110 structural responses were measured and recorded per accelerometer. We randomly selected the repeated test results from the same accelerometer under the same damage scenario as either training or testing data.

Typical structural responses from Accelerometer 1 under intact scenario, damage scenario 1, and damage scenario 10 are shown in Figure 5. A comparison between two repeated

Table 2. Damage scenarios

\begin{tabular}{|c|c|c|}
\hline Description & $\begin{array}{l}\text { Damage } \\
\text { scenario }\end{array}$ & $\begin{array}{l}\text { Bolts } \\
\text { loosened }\end{array}$ \\
\hline Intact & 0 & All tight \\
\hline \multirow{3}{*}{$\begin{array}{l}\text { One bolt loosened at one } \\
\text { end at different locations }\end{array}$} & 1 & 1 \\
\hline & 2 & 3 \\
\hline & 3 & 2 \\
\hline \multirow{3}{*}{$\begin{array}{l}\text { Two bolts loosened at one } \\
\text { end at different locations }\end{array}$} & 4 & 1 and 2 \\
\hline & 5 & 1 and 3 \\
\hline & 6 & 1 and 4 \\
\hline \multirow{2}{*}{$\begin{array}{l}\text { Two bolts loosened at both } \\
\text { ends at different locations }\end{array}$} & 7 & $1,2,5$ and 6 \\
\hline & 8 & $1,3,5$, and 7 \\
\hline Three bolts loosened at one end & 9 & 1,2, and 3 \\
\hline Four bolts loosened at one end & 10 & $1,2,3$ and 4 \\
\hline
\end{tabular}

Figure 6. Comparison of training data and testing data

test results for the intact scenario, i.e. a training data example and a testing data example, is shown in Figure 6. The results from other accelerometers are similar.

As can be seen, the difference between the intact scenario and damage scenarios is mainly the amplitude, while it is 


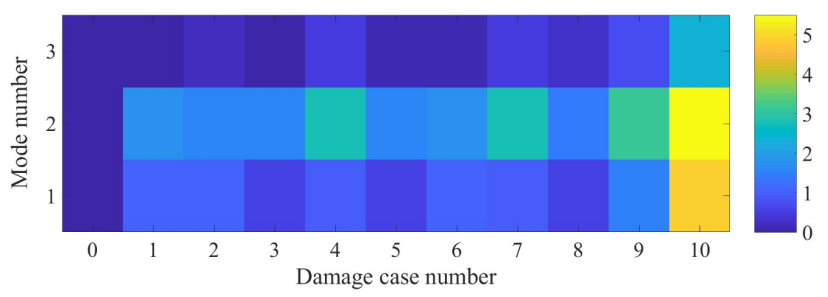

Figure 7. Percentile change in frequencies for different damage scenarios

hard to differentiate the damage scenarios. From intuitive comparison, the difference between data from two different damage scenarios is comparable to that between two randomly selected data sets (as training and testing) in one scenario. Therefore, without the analysis of these signals, it is almost impossible to identify structural conditions.

\section{Modal identification results}

In order to verify the performance of the traditional methods, modal parameters, i.e. natural frequencies, mode shapes, and damping ratios, need to be extracted. The Welch method is used to develop an in-house MATLAB program for modal identification.

Based on the test data from all the six accelerometers, the modal identification results for all the damage scenarios were obtained from the average of these ten repeated tests. As shown in Table 3 and Figure 7, it is clear that the average change in frequency for the first nine damage cases is less than $2 \%$, while it is $4.05 \%$ for damage case 10 when all the four bolts are loosened at one end of the column to beam joint. Since the average change in frequency is less than $2 \%$, it is very difficult to identify damage for the first 9 damage cases. This is aligned with Salawu (1997), which reported that the identification of small damage may not be possible by using changes in frequency data alone.

The changes in mode shape based parameters were expected to be more efficient in identifying structural damage, however it is evident from Figure 8 that the mode shapes from the first nine damage scenarios are almost identical to that of the intact structure. For damage scenario

Table 3. Identified natural frequencies and modal damping raitos

\begin{tabular}{|c|c|c|c|c|c|c|}
\hline \multirow{2}{*}{$\begin{array}{c}\text { Damage } \\
\text { scenario }\end{array}$} & \multicolumn{3}{|c|}{ Natural frequencies } & \multicolumn{3}{c|}{ Damping ratios (\%) } \\
\cline { 2 - 7 } & 1st & 2nd & 3rd & 1st & 2nd & 3rd \\
\hline 0 & 82.43 & 258.80 & 563.20 & 0.15 & 0.18 & 0.16 \\
\hline 1 & 83.34 & 254.30 & 563.30 & 0.15 & 0.44 & 0.18 \\
\hline 2 & 83.34 & 254.70 & 564.60 & 0.16 & 0.47 & 0.14 \\
\hline 3 & 82.92 & 254.70 & 563.30 & 0.16 & 0.50 & 0.17 \\
\hline 4 & 81.63 & 251.70 & 560.70 & 0.17 & 0.52 & 0.20 \\
\hline 5 & 82.92 & 254.70 & 562.50 & 0.16 & 0.49 & 0.18 \\
\hline 6 & 83.34 & 254.30 & 563.70 & 0.15 & 0.44 & 0.18 \\
\hline 7 & 81.63 & 251.70 & 560.70 & 0.16 & 0.48 & 0.16 \\
\hline 8 & 82.92 & 255.20 & 561.60 & 0.17 & 0.48 & 0.21 \\
\hline 9 & 81.21 & 250.90 & 559.00 & 0.17 & 0.73 & 0.22 \\
\hline 10 & 78.64 & 245.30 & 550.10 & 0.89 & 2.11 & 0.23 \\
\hline
\end{tabular}

10, although small changes in all three mode shapes can be identified, these changes occur all along the length of beam, which contradicts the fact that the actual damage is present only at one end connection.

The modal assurance criterion (MAC) is commonly used to measure the similarity between two mode shapes. A MAC value of 1 indicates that the two mode shapes are identical. A damage in the structure is expected to change the mode shape and a change in MAC value. However, from Figure 9, it is clear that the change in MAC value is evident only in damage scenario 10, while these are almost identical in other damage scenarios. Thus, a change in MAC based technique may be used to identify only one damage scenario while it fails to identify the other nine damage scenarios. Therefore, the mode shape based methods may only be able to detect the damage with all bolts loosened, but will struggle in identifying (locating and quantifying) this kind of damage at the end boundary conditions.

From Table 3 and Figure 10, the changes in modal damping ratios seem to be a promising parameter to differentiate damage scenarios. Particularly, the damping ratio of the second mode changed $144 \%$ between the intact structure and the first damage scenario; the change became 11.72 times when considering all bolts loosened damage scenarios. These results are aligned with Ay et al. (2019), which showed the superior sensitivity of damping parameters to damage. However, Ay et al. (2019) used a parameter describing the overall damping effect, while in this case, only the damping ratio of the second mode is sensitive to the damage. So the direct application of Ay et al. (2019) may not be feasible for this study.

Based on the modal identification results, it is clear that the traditional methods using natural frequencies and mode shapes are not able to detect the damage unless all bolts are loosened at a connection. Although the damping parameters has the potential to be a useful damage indicator, the understanding and the measurement of damping, plus the generation of damping related indicators, are still challenging. Therefore, further studies are needed to correlate various damage parameters to the changes in damping parameters, which is out of the scope of this study.

\section{Results and discussions}

\section{Implementation details}

In this work, we normalised the acquired acceleration data according to input force. Firstly, we re-scaled the impact loading to a unit value by multiplying a scaling factor for each strike, and then multiplied the same scaling factor to the acceleration responses for all the six accelerometers. In order to reduce the signal noise level, we secondly apply a band-pass filter to the data $(50 \mathrm{~Hz}$ to $600 \mathrm{~Hz}$, to cover all three modes according the modal analysis results). Thirdly, we re-sampled the data to 5000 points each (equivalent to a sampling rate of $2048 \mathrm{~Hz}$ ). Finally, we labelled the data from different accelerometers under different scenarios with its associated scenario numbers, which were ready to be used for network training.

The normalised data sets and their associated labels were used to train the SHMnet for the following four parametric studies. The trained SHMnet model was then used to identify 

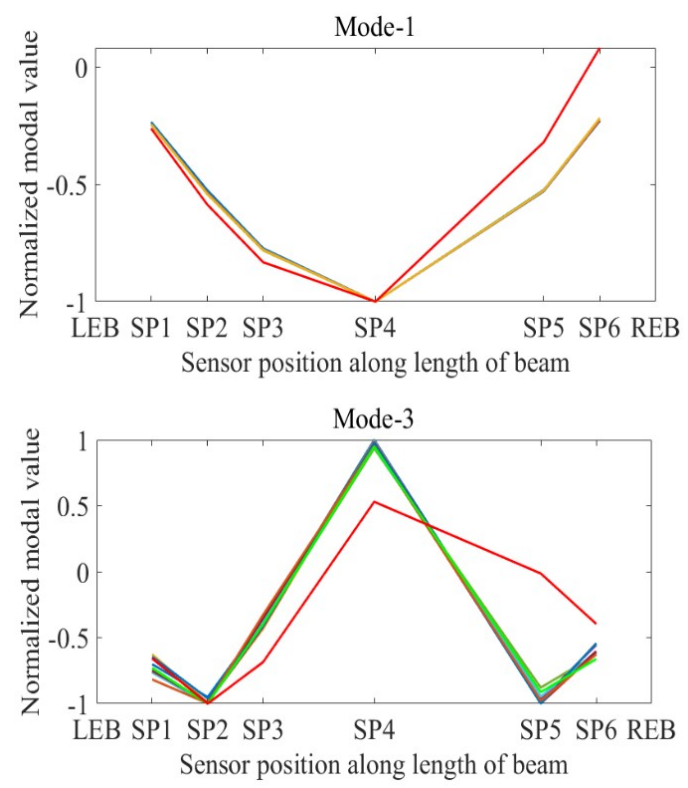

Mode-2

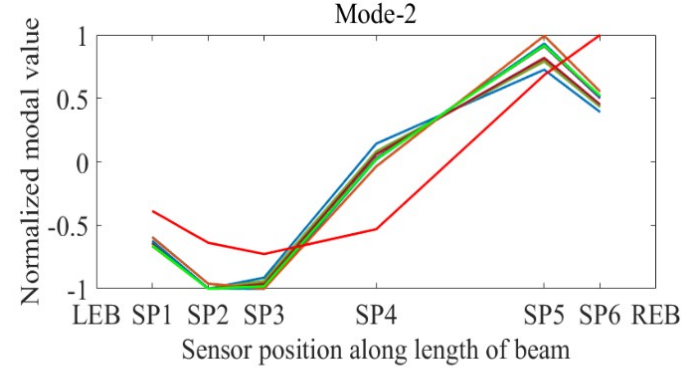

- Damage case - 0 - Damage case - 6
- Damage case - 1 - Damage case - 7
- Damage case - 2 - Damage case - 8
- Damage case - 3 - Damage case - 9
- Damage case - 4 - Damage case - 10
- Damage case - 5

Figure 8. Mode shapes for different damage scenarios
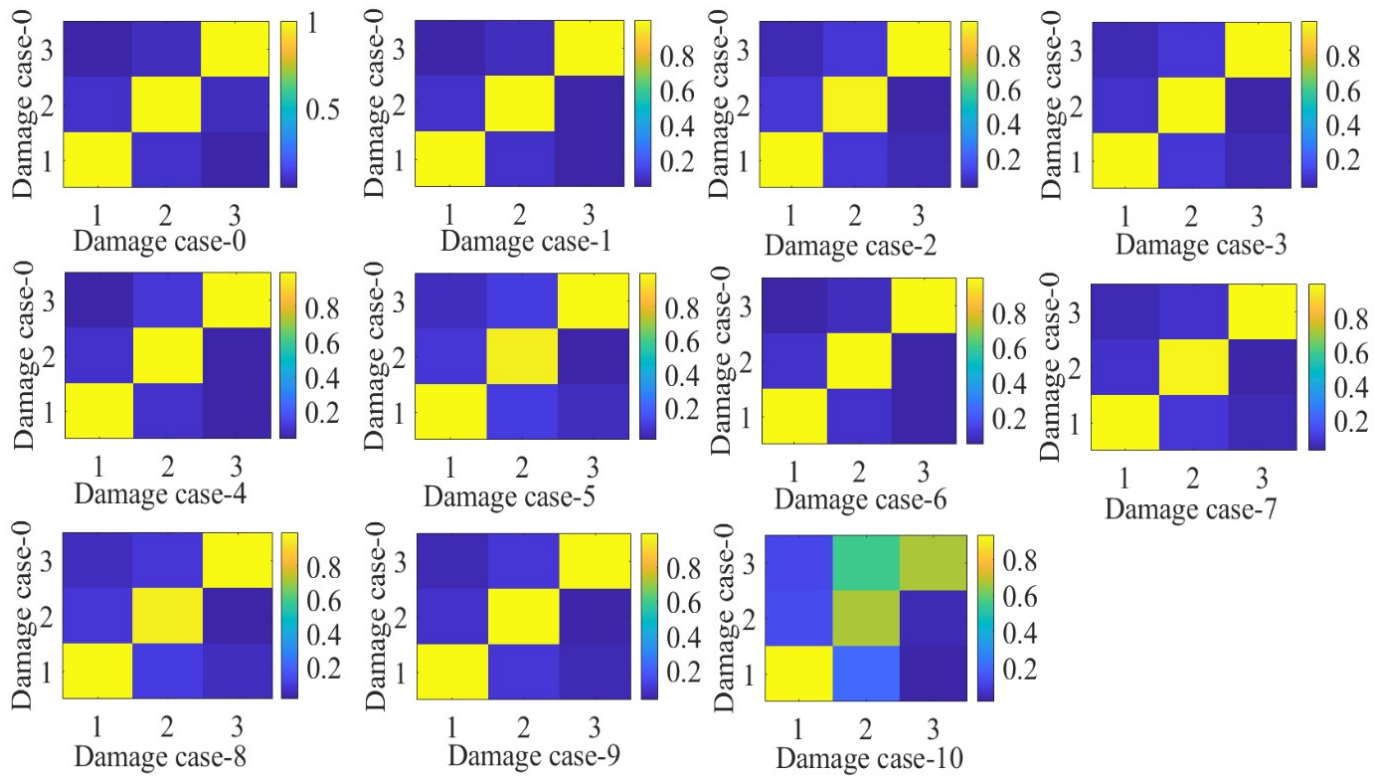

Damage case-7

Figure 9. MAC matrices comparison between the intact structure and different damage scenarios

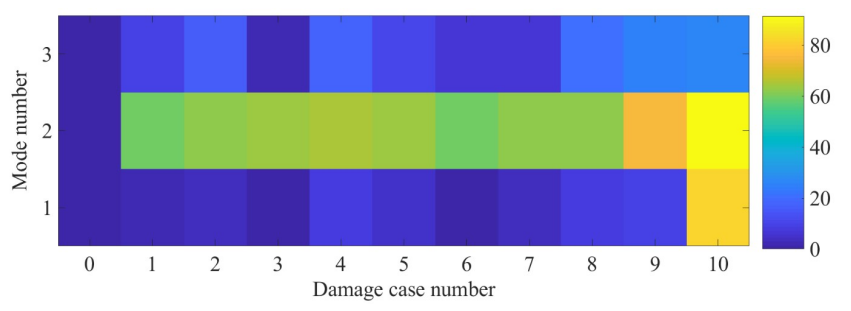

Figure 10. Percentile change in modal damping ratios for different damage scenarios

the structural condition label with "unknown" scenarios by using the randomly selected testing data sets. If the correct label was found, we considered the result is accurate, and vice versa. We evaluate the accuracy of the SHMnet model on all the 11 scenarios with five testing data sets for each scenario.

\section{Effect of data augmentation scheme}

It is common to use data augmentation schemes in deep learning algorithms, to consider a variety of conditions but with only limited training data. Different data augmentation schemes are available, but most are only feasible for images. Gaussian noise, which has zero mean value, essentially has data points at all frequencies, and is expected to be able to enhance the learning capability. Therefore, to examine the effects of data augmentation schemes, the first parametric study focuses on the effects of adding Gaussian noise at different levels to the original training data. We compared the structural condition identification results by using the original training data, with those smeared with $10 \%$ and $20 \%$ Gaussian noise. The training data were from Accelerometer 1. Our previous study (Zhang and Wang (2019)) showed that at least four repeated test results will be needed for reliable results. Considering this case being more challenging, we 


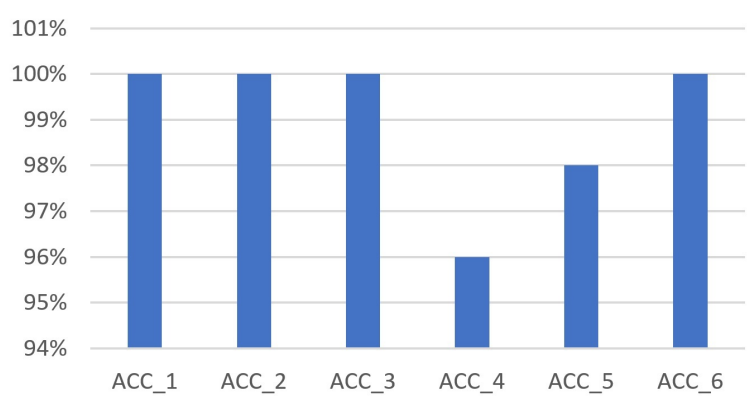

Figure 11. Comparison of identification accuracy at different sensor locations

used five repeated test results as training data while the rest five as testing data.

The condition identification accuracy results are shown in Table 4. It can be seen that without data augmentation, the algorithm can achieve $98 \%$ accuracy, which is already very good. With either $10 \%$ or $20 \%$ noise, the accuracy is enhanced to $100 \%$. It means that with data augmentation in place, the accuracy can improve, but it is not sensitive to the noise level. Therefore, in the rest of this study, we use $10 \%$ Gaussian noise for data augmentation.

\section{Effect of sensor locations}

Next, we examine the effects of sensor locations on the accuracy of the proposed algorithm. We used five repeated test data from six accelerometers as six groups of training data. With each training data group, an SHMnet model will be trained and used for condition identification.

The accuracy results of using sensor data at different locations are shown in Figure 11. It can bee seen that the algorithm can achieve $100 \%$ identification accuracy by using sensor data from Accelerometers 1, 2, 3, and 6. Even by using Accelerometers 4 and 5, 96\% and 98\% accuracy levels can be achieved. Accelerometer 4 is at the middle of the beam, which essentially loses all the information from the even number modes. As shown in Modal identification results, the second mode is actually the most sensitive mode. Therefore, the declining of accuracy is expectable. Regarding Accelerometer 5, the accuracy degrades because 1) it is at the nodal point of a higher mode ( $1 / 5$ of the total beam length); 2) the location is far from the impact point, and thus the vibration amplitude is much lower than its symmetrical location, Acceleromter 2.

The results can serve a preliminary guide for the researchers in this field to select sensor locations. Firstly, the nodal points of vibration modes should be avoided. Secondly, the distance between the accelerometer and the impact hammer may slightly affect identification results. Indeed, if the nodal points have been avoided, the results are not sensitive to the actual sensor location.

Table 4. Comparison testing results with different training data augmentation with addictive random Gaussian noise

\begin{tabular}{c|c|c|c}
\hline Noise level & 0 & $10 \%$ & $20 \%$ \\
\hline Accuracy & $98 \%$ & $100 \%$ & $100 \%$ \\
\hline
\end{tabular}

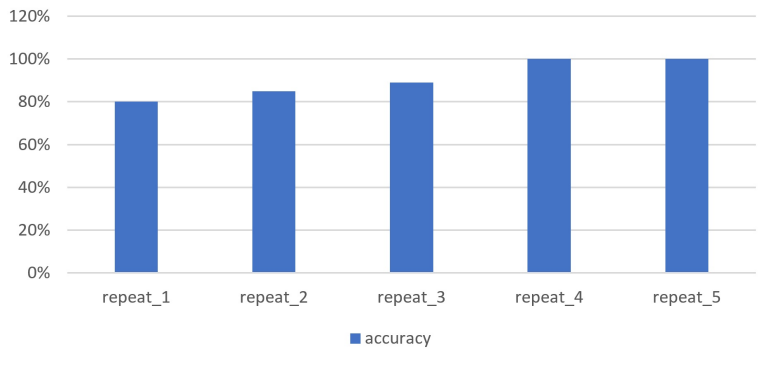

Figure 12. Condition identification accuracy using different number of training data at Accelerometer 1

In the rest parametric studies, we select the data from Accelerometer 1 as the training data.

\section{Effect of the number of training data sets}

To examine how many training data sets can deliver reliable networks for structural identification, we trained the SHMnet for five times, with an increasing number of experimental data sets as training data, i.e. $1,2,3,4$, and 5 . The rest five repeated test data were used as testing data. Figure 12 shows the condition identification accuracy results using different number of training data sets from Accelerometer 1. It clearly shows that with just one test data as training, the structural identification accuracy can achieve $80 \%$. This is extraordinary because the normal physics-based methods require at least four to six repeated test results from several acceleometers to extract reliable modal parameters.

With the increase of training data set number, the identification accuracy increase dramatically. With at least four repeated test data as training, the proposed SHMnet can achieve $100 \%$ condition identification accuracy, which is coincident with the results from Zhang and Wang (2019). This may suggest that for future studies, at least four repeated tests are necessary to train the SHMnet model, which can achieve optimal condition identification results.

\section{Robustness of the algorithm}

The last parametric study in this work focuses on the robustness of the proposed algorithm. The SHMnet was firstly trained by using five repeated test data from Accelerometer 1 under 11 scenarios. We then added different levels of Gaussian noise onto the testing data and used the trained model to identify/classify structural conditions. We illustrate the difference between the original test data and that with different noise levels in Figure 13. It can be seen that with $50 \%$ noise, the vibration data become clearly different, while $10 \%$ noise does not change the original signal much.

To obtain reliable results, we performed 30 repeated tests on the structural identification using the data with $20 \%, 40 \%$, $60 \%, 80 \%$, and $100 \%$ Gaussian noises. The statistical results are summarised in Table 5. It clearly shows that the structural condition identification using SHMnet is reliable when the test data are smeared with $60 \%$ noise, with over $98 \%$ percent accuracy and $0.37 \%$ standard deviation. Even with $100 \%$ noise, the accuracy can still achieve more than $85 \%$. 


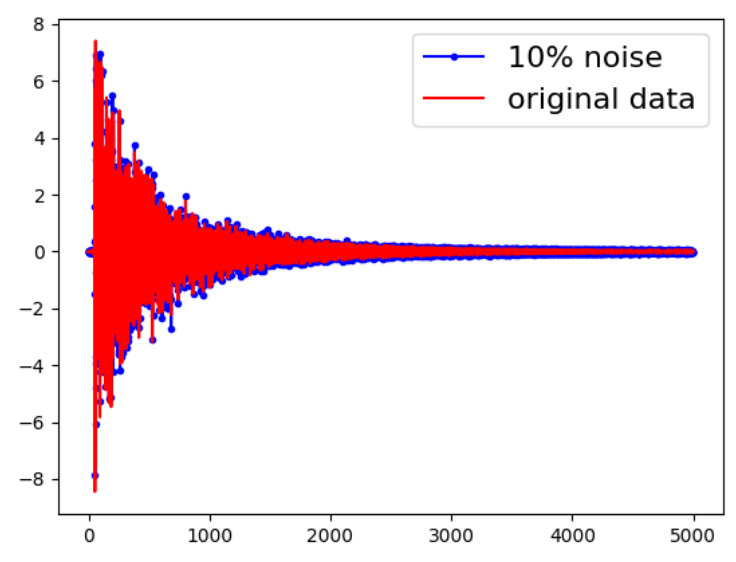

(a)

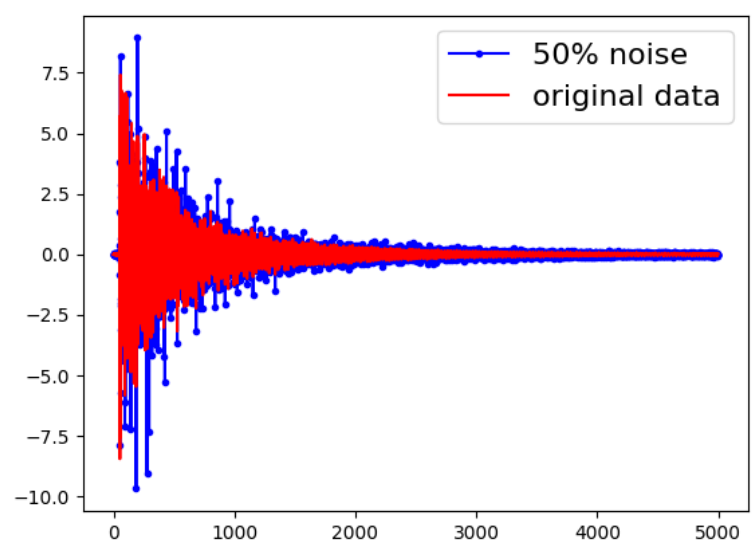

(b)

Figure 13. Illustration of the original data and the data with different noise levels: (a) $10 \%$; (b) $50 \%$

Table 5. Comparison average results with $20 \%$ to $100 \%$ random Gaussian noise added to the testing data with 30 repeated tests

\begin{tabular}{c|c|c|c|c|c}
\hline Noise level & $20 \%$ & $40 \%$ & $60 \%$ & $80 \%$ & $100 \%$ \\
\hline Mean $\pm S t d$ & $100 \% \pm 0$ & $99.23 \% \pm 0.42 \%$ & $98.17 \% \pm 0.37 \%$ & $93.33 \% \pm 0.60 \%$ & $85.83 \% \pm 0.82 \%$ \\
\hline
\end{tabular}

\section{Discussions}

Comparison between SHMnet and traditional methods: Comparing the results by using SHMnet and those from traditional modal identification, the effectiveness and robustness of the proposed method can be easily recognised. Especially, the overall accuracy of the proposed method by using four repeated test data from only one accelerometer can achieve $100 \%$, and it is over $98 \%$ even by adding $60 \%$ Gaussian noise to the testing data. In contrast, only the damping ratio of the second vibration mode can indicate a certain level of changes, while all the other existing damage indicators, based on natural frequencies, mode shapes, and MAC, fail to detect the conditions under scenarios 1-9. Only damage scenario 10 , i.e. all bolts at a joint being loosened, can be successfully detected.

Data preparation scheme: The first three parametric studies confirm that the quantity and quality of training data affect the performance of a data-driven algorithm. In SHM domain, the data augmentation scheme using different Gaussian noise levels is insensitive to the performance of the algorithm, while, without data augmentation, the identification performance will slightly degrade. So it is suggested to add a standard Gaussian noise for data augmentation.

The sensor locations may affect the algorithm performance to a larger degree. In this case, the worst case scenario is Accelerometer 4, which is located at a vibration node point. This kind of locations need to be avoided for the better performance of a data-driven algorithm. The existing optimal sensor location methods usually focus on the physics-based method, i.e. the extraction of modal parameters. Therefore, further studies are needed to optimise the sensor locations which suit for the data-driven algorithms.

The number of repeated impact hammer tests is a very important factor for determining the algorithm performance, but it was rarely discussed in existing studies. Based on this study and Zhang and Wang (2019), if we aim 100\% condition identification accuracy, at least four repeated tests are needed. But at well selected sensor locations, even one test data may lead to an acceptable accuracy (80\% in this work).

Computational efficiency: The experiments were conducted using a computer with I7-8700 CPU at $3.2 \mathrm{GHz}(12$ cores) and 16-Gb memory. NVidia Titan XP GPU with 12$\mathrm{Gb}$ memory is used to accelerate the computation. For the following parametric studies, the average training time for SHMnet is 1500 s with 1000 epochs. For a single test data, the classification time is $9.3 \mathrm{~ms}$.

\section{Conclusions}

This paper proposed a novel structural condition identification framework, i.e., SHMnet, based on a popular deep learning network architecture Alex-net. The influencing factors on the performance of SHMnet were studied through a case study i.e., a steel frame with bolt connection damage. We found out that the performance not only depends on the network architecture, but also relies on the quality and quantity of the training data. In the laboratory, a steel frame was constructed and impact hammer tests were performed. Ten damage scenarios together with an intact scenario were designed to test the performance of the algorithm under such subtle structural condition change as one bolt loosened. The structural condition identification results demonstrate:

1) The proposed SHMnet is very effective in structural condition identification, with $100 \%$ identification accuracy using at least four repeated test data from just one accelerometer as training. In contrast, the traditional modalbased methods fail in this case, as the identified modal parameters under intact scenario are very close to those under damage scenarios except all bolts being loosened.

2) Among the three influencing factors considered in this study, the quantity of the training data sets is very important. 
Four is a recommended number for repeated tests, aiming to a $100 \%$ identification accuracy. The sensor location is another factor that may influence the algorithm performance. If an accelerometer is at vibration node points of a structure, the identification accuracy inevitably degrades. The closer distance between accelerometer and impact may also benefit the identification accuracy. In contrast, the popular data augmentation schemes with different levels of Gaussian noise are insensitive to the algorithm accuracy. A $10 \%$ Gaussian is recommended based on the results from this study.

3) The proposed SHMnet is robust when the testing data are smeared with Gaussian noise. Even under $60 \%$ noise level, the structural condition identification can achieve the accuracy of over $98 \%$.

Overall, our results demonstrate the potential of the proposed SHMnet as an effective structural condition identification tool for SHM. The future efforts may be placed at: 1) optimising the sensor locations for data-driven SHM methods; 2) testing the performance of SHMnet under different signal types, e.g. ambient vibration; 3) generating more training data from numerical simulation or generative adversarial networks.

\section{Acknowledgement}

The authors would like to thank the Engineering and Physical Science Research Council (EPSRC), UK, for the financial support through Grant EP/R021090/1. The authors also thank NVidia for the GPU grant.

\section{References}

Abdeljaber O, Avci O, Kiranyaz MS, Boashash B, Sodano H and Inman DJ (2018) 1-d cnns for structural damage detection: verification on a structural health monitoring benchmark data. Neurocomputing 275: 1308-1317.

Abdeljaber O, Avci O, Kiranyaz S, Gabbouj M and Inman DJ (2017) Real-time vibration-based structural damage detection using one-dimensional convolutional neural networks. Journal of Sound and Vibration 388: 154-170.

Ay AM, Khoo S and Wang Y (2019) Probability distribution of decay rate: a statistical time-domain damping parameter for structural damage identification. Structural Health Monitoring 18(1): 66-86.

Bao Y, Tang Z, Li H and Zhang Y (2018) Computer vision and deep learningbased data anomaly detection method for structural health monitoring. Structural Health Monitoring: An International Journal DOI:10.1177/1475921718757405.

Biswal S and Wang Y (2019) Optimal sensor placement strategy for the identification of local bolted connection failures in steel structures. In: Proceedings of the 2019 International Conference on Smart Infrastructure and Construction (ICSIC 2019). ICE Publishing.

Blachowski B and Gutkowski W (2016) Effect of damaged circular flange-bolted connections on behaviour of tall towers, modelled by multilevel substructuring. Engineering Structures 111: 93-103.

Cha YJ, Choi W and Büyüköztürk O (2017) Deep learning-based crack damage detection using convolutional neural networks.
Computer-Aided Civil and Infrastructure Engineering 32(5): 361-378.

Cha YJ, You K and Choi W (2016) Vision-based detection of loosened bolts using the hough transform and support vector machines. Automation in Construction 71: 181-188.

Ewins D (2000) Modal testing: theory, practice and application .

Farrar C and Worden K (2013) Structural Health Monitoring: A Machine Learning Perspective. ISBN 978-1-119-99433-6. DOI:10.1002/9781118443118.

He K and Zhu W (2011) Structural damage detection using changes in natural frequencies: theory and applications. In: Journal of Physics: Conference Series, volume 305. IOP Publishing, p. 012054.

Kingma DP and Ba J (2014) Adam: A method for stochastic optimization. arXiv preprint arXiv:1412.6980 .

Krizhevsky A, Sutskever I and Hinton GE (2012) Imagenet classification with deep convolutional neural networks. In: Advances in neural information processing systems. pp. 10971105.

Lacey AW, Chen W, Hao H and Bi K (2018) Structural response of modular buildings-an overview. Journal of Building Engineering 16: 45-56.

LeCun YA, Bengio Y and Hinton GE (2015) Deep learning. Nature DOI: $10.1038 /$ nature 14539.

Li J and Hao H (2016) Health monitoring of joint conditions in steel truss bridges with relative displacement sensors. Measurement 88: 360-371.

Nair V and Hinton GE (2010) Rectified linear units improve restricted boltzmann machines. In: Proceedings of the 27th international conference on machine learning (ICML-10). pp. 807-814.

Pathirage CSN, Li J, Li L, Hao H, Liu W and Ni P (2018) Structural damage identification based on autoencoder neural networks and deep learning. Engineering Structures DOI: 10.1016/j.engstruct.2018.05.109.

Pathirage CSN, Li J, Li L, Hao H, Liu W and Wang R (2019) Development and application of a deep learning-based sparse autoencoder framework for structural damage identification. Structural Health Monitoring 18(1): 103-122.

Salawu O (1997) Detection of structural damage through changes in frequency: a review. Engineering structures 19(9): 718-723.

Shao J, Wang T, Yin H, Yang D and Li Y (2016) Bolt looseness detection based on piezoelectric impedance frequency shift. Applied Sciences 6(10): 298.

Silver D, Huang A, Maddison CJ, Guez A, Sifre L, Van Den Driessche G, Schrittwieser J, Antonoglou I, Panneershelvam V, Lanctot M, Dieleman S, Grewe D, Nham J, Kalchbrenner N, Sutskever I, Lillicrap T, Leach M, Kavukcuoglu K, Graepel $\mathrm{T}$ and Hassabis D (2016) Mastering the game of Go with deep neural networks and tree search. Nature DOI:10.1038/ nature16961.

Srivastava N, Hinton G, Krizhevsky A, Sutskever I and Salakhutdinov R (2014) Dropout: a simple way to prevent neural networks from overfitting. The Journal of Machine Learning Research 15(1): 1929-1958.

Wang F, Huo L and Song G (2017) A piezoelectric active sensing method for quantitative monitoring of bolt loosening using energy dissipation caused by tangential damping based on the fractal contact theory. Smart Materials and Structures 27(1): 
015023.

Wang T, Song G, Wang Z and Li Y (2013) Proof-of-concept study of monitoring bolt connection status using a piezoelectric based active sensing method. Smart Materials and Structures 22(8): 087001.

Wang Y and Hao H (2013) Damage identification scheme based on compressive sensing. Journal of Computing in Civil Engineering 29(2): 04014037.

Yang JN, Xia Y and Loh CH (2013) Damage identification of bolt connections in a steel frame. Journal of Structural Engineering 140(3): 04013064.

Yun CB, Yi JH and Bahng EY (2001) Joint damage assessment of framed structures using a neural networks technique. Engineering structures 23(5): 425-435.

Zhang T and Wang Y (2019) Deep learning algorithms for structural condition identification with limited monitoring data. In: Proceedings of the 2019 International Conference on Smart Infrastructure and Construction (ICSIC 2019). ICE Publishing.

Zhang Y, Sun X, Loh KJ, Su W, Xue Z and Zhao X (2019) Autonomous bolt loosening detection using deep learning. Structural Health Monitoring : 1475921719837509. 